\title{
RAPID ACQUISITION OF ULTRA-WIDEBAND SIGNALS IN THE DENSE MULTIPATH CHANNEL
}

\author{
Eric A. Homier and Robert A. Scholtz
}

\begin{abstract}
Efficient serial search strategies are presented and shown to drastically reduce the mean acquisition time for UWB signals in a dense multipath environment. Inherent in traditional serial search problems is the assumption that only a single bin or a small number of consecutive bins will properly terminate the search. This assumption leads to search strategies which tend to be linear in nature, e.g., a linear sweep of the uncertainty region. Because of the dense multipath channel present in most UWB systems this assumption is invalid as seen by the channel's relatively large delay spread. A generalized analysis of various search algorithms is presented based upon a Markov chain model of a simple singledwell serial search. The results from this analysis reveal that the linear search has a considerably larger mean acquisition time than the more efficient search strategy termed the bit reversal search.
\end{abstract}

\section{INTRODUCTION}

An ultra-wideband (UWB) signal is one with a fractional bandwidth, $B_{f}=2\left(f_{H}-f_{L}\right) /\left(f_{H}+f_{L}\right)$, greater than 25 percent [1]. Here $f_{L}$ and $f_{H}$ are the lower and upper end ( $3 \mathrm{~dB}$ points) of the signal spectrum. Ultra-wideband signals are currently being investigated for use in communications systems where an advantage over more narrowband signals exists. These advantages include improved penetration through materials as well as improved performance in dense multipath environments [2] where the UWB signals can be resolved in time making the use of a RAKE receiver possible. These advantages make UWB communication systems well suited for urban and indoor wireless applications.

The specific UWB signal and the dense multipath channel model are defined in the next section. Then the output of a simple, single correlator receiver model is investigated, followed by a Markov analysis of the acquisition process. Specific search algorithms are examined for the ideal case of no noise and it is shown that the bit reversal search is the optimum strategy among the group. Finally, the straight linear search and the bit reversal search are compared for an actual indoor multipath channel. The results prove that the bit reversal search

Eric Homier is with TRW Space \& Electronics in Redondo Beach, California (homier@usc.edu). Robert Scholtz is Prof. of Electrical Engineering with the Univ. of Southern California (scholtz@usc.edu). This work was supported in part by the Department of Defense under MURI Grant No. DAAD19-01-1-0477 and in part by a TRW Doctoral Fellowship. decreases considerably the expected number of observations required to complete the search.

\section{Signal And Multipath Models}

The signal choice for the UWB signal in this paper is a baseband pulse that is shaped as the $2^{\text {nd }}$ derivative of a Guassian pulse:

$$
p(t)=\sqrt{\frac{4}{3 \sigma \sqrt{\pi}}}\left(1-\left(\frac{t}{\sigma}\right)^{2}\right) \exp \left(-\frac{1}{2}\left(\frac{t}{\sigma}\right)^{2}\right)
$$

The energy in $p(t)$ is unity so that the received energy in $\sqrt{E_{p}} \cdot p(t)$ is simply $E_{p}$. The scale factor, $\sigma$, determines the effective time width of the pulse shape and will be considered approximately $(2 \sqrt{\pi})^{-1} \cdot 1$ nsec. This UWB pulse is then repeated once per frame time which yields the following unmodulated signal:

$$
x(t)=\sqrt{E_{p}} \cdot \sum_{n} p\left(t-n T_{f}\right)
$$

Here $T_{f}$ is the frame time at the transmitter. One possible modulation for a multiple user system is pulse position modulation in which each user has a specific time-hopping code [3].

The multipath channel to be considered in this paper assumes a specular model with an impulse response

$$
h(t)=\sum_{m=0}^{M-1} a_{m} \delta\left(t-\tau_{m}\right)
$$

For the purposes of this paper, the amplitude coefficients, $a_{m}$, and the time delays, $\tau_{m}$, are determined using the CLEAN algorithm, a form of subtractive deconvolution, on a measured UWB signal in an indoor office environment [4]. Measured and reconstructed waveforms are shown in Figure 1 for $M=300$ where the amplitude coefficients are normalized so that $\sum_{m} a_{m}^{2}=1$. Only the relative path delays, defined as $\Delta \tau_{m}=\tau_{m}-\tau_{0}$ for $m=0,1, \cdots, M-1$, will be known and the absolute value of $\tau_{0}$ with respect to the receiver's clocks will be assumed uniform over the frame time. The goal of the acquisition process discussed here is to provide proper frame alignment, in essence accounting for the random delay $\tau_{0}$. 


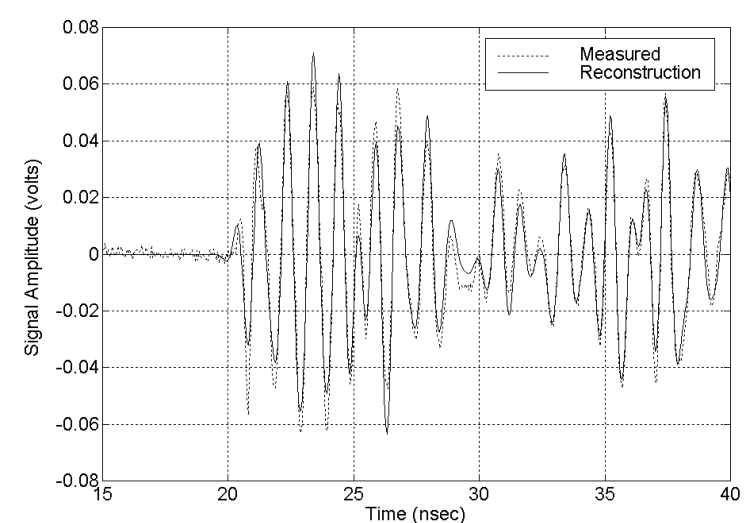

Fig. 1. Pulse response function of an indoor multipath channel and reconstructed waveform

The received signal at the multipath channel output, with AWGN $n(t)$, is

$$
s(t)=\sqrt{E_{p}} \sum_{n=0}^{\infty} \sum_{k=0}^{M-1} a_{k} p\left(t-n T_{f}-\tau_{k}\right)+n(t)
$$

The transmitter frame time, $T_{f}$, is assumed known at the receiver. Thus, the local correlator will have the template waveform

$$
v(t)=\sum_{m=0}^{\infty} p\left(t-m T_{f}-\varepsilon_{(m \bmod N)}\right)
$$

The search location, $\varepsilon_{n}$ for $n=0,1, \cdots, N-1$, changes every frame time and represents the time bin center currently being searched where the bin spacing is fixed at $T_{f} / N$. Different search algorithms, which are simply different permutations of the bin search order, are to be examined below for a single correlator receiver. A simple acquisition algorithm for multiple correlators as in a selective RAKE receiver would be to divide the search among the correlators as follows: if the search locations are $\varepsilon_{0}, \varepsilon_{1}, \varepsilon_{2}$, etc. and there are $L_{p}$ correlators available then the first correlator would search $\varepsilon_{0}, \varepsilon_{L_{p}}, \varepsilon_{2 L_{p}}, \ldots$, the second correlator would search $\varepsilon_{1}, \varepsilon_{L_{p}+1}, \varepsilon_{2 L_{p}+1}, \ldots$ and so forth. The mean search time should decrease by a factor of $L_{p}$ when employing this approach.

\section{Correlator Output}

Only a single correlator receiver structure will be considered here. The correlator output for the $j^{\text {th }}$ frame, $z_{j}$, has a signal and noise component, $s_{j}$ and $n_{j}$ respectively. The mean of the AWGN noise process $n(t)$ is zero and the autocorrelation is simply $N_{0} \delta\left(t_{1}-t_{2}\right)$. In the following expressions, the substituted variable $\theta_{j}=j T_{f}+\varepsilon_{(j \bmod N)}$ is used. The noise component is now computed as

$$
n_{j}=\int_{\theta_{j}-\Delta}^{\theta_{j}+\Delta} n(t) v(t) \mathrm{dt}
$$

Here the pulse shape is assumed to span only $-\Delta$ to $+\Delta$. Since we are only integrating over one portion of a frame and because the frames do not overlap we see that the output noise sequence is independent. The mean and variance of $n_{j}$ are computed as 0 and $N_{0}$, respectively.

The signal component, $s_{j}$, of the correlator is fairly straightforward to compute after several simplifying assumptions are made. First, the receiver frame time will be assumed long enough with respect to the 'delay spread' of the multipath channel so that only energy from the $(m-1)^{\text {st }}$ frame will potentially spill into the $m^{\text {th }}$ frame. It is also assumed that $\varepsilon_{(m \bmod N)}$ only varies over the $m^{\text {th }}$ frame at the receiver. These assumptions result in the following expression, which is written explicitly as a function of $\tau_{0}$ :

$$
\begin{array}{r}
s_{j}\left(\tau_{0}\right)=\sqrt{E_{p}} \sum_{k} a_{k} \cdot\left[\gamma\left(\tau_{0}+\Delta \tau_{k}-\varepsilon_{(j \bmod N)}\right)\right. \\
\left.+\gamma\left(\tau_{0}+\Delta \tau_{k}-\varepsilon_{(j \bmod N)}-T_{f}\right)\right]
\end{array}
$$

The function $\gamma(\tau)$ is the pulse autocorrelation function of $p(t)$ in (1). This can be shown to equal

$\gamma(\tau)=\left(1-\left(\frac{\tau}{\sigma}\right)^{2}+\frac{1}{12} \cdot\left(\frac{\tau}{\sigma}\right)^{4}\right) \exp \left(-\frac{1}{4} \cdot\left(\frac{\tau}{\sigma}\right)^{2}\right)$

\section{Markov Analysis of UWB Acquisition}

A general framework, based upon a Markov chain model, will now be used to derive the results for the expected search time of a simple single-dwell serial search acquisition scheme. This scheme terminates the first time the correlator magnitude exceeds some prescribed threshold, say $\sqrt{E_{p}} \Upsilon$ where $\Upsilon$ is some normalized threshold. In this simple acquisition scheme the false alarm and acquisition states are the same state. Usually a verification phase is required to detect false alarms and thus yields independent false alarm and acquisition states. For the purposes of this paper, however, no verification phase is used so that the chain in Figure 2 represents the acquisition scheme. The transition probabilities are seen to be

$$
p_{n}=\operatorname{Pr}\left(\left|z_{j}\right| \leq \sqrt{E_{p}} \Upsilon\right) \text { for } j=0,1,2, \cdots
$$

Here $n=j \bmod N$ is the Markov chain state number and $j$ is the frame number. Each state in the chain represents a particular search area. State $N$ represents that the search has terminated and is $a b$ sorbing. 


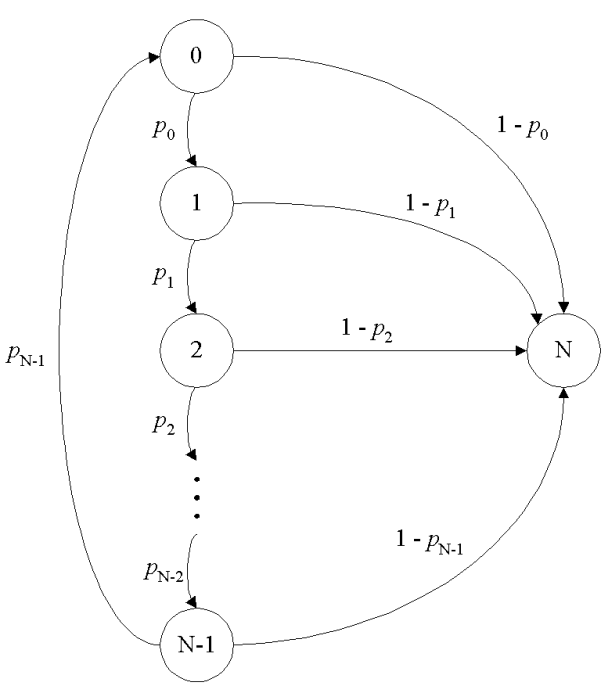

Fig. 2. Markov chain model for the serial search

Since state $N$ is the only recurrent state of the chain and represents the fact that the search has terminated, the expected search time can be computed by finding the expected time it takes to enter this recurrent state, denoted $E\left(T_{r}\right)$. For the chain of Figure 2 this mean time is (in number of states visited):

$$
E\left(T_{r}\right)=\frac{1+\sum_{m=0}^{N-2} \prod_{n=0}^{m} p_{n}}{1-\prod_{n=0}^{N-1} p_{n}}
$$

For the correlator output as given in section III the transition probabilities, $p_{n}=p_{(j \bmod N)}=\operatorname{Pr}\left(\left|z_{j}\right| \leq\right.$ $\left.\sqrt{E_{p}} \Upsilon\right)$, are seen to be functions of $\tau_{0}$ and can be found as

$$
\begin{aligned}
p_{n}\left(\tau_{0}\right)= & 1-Q\left(\sqrt{\frac{E_{p}}{N_{0}}}\left(\Upsilon+\frac{s_{j}\left(\tau_{0}\right)}{\sqrt{E_{p}}}\right)\right) \\
& -Q\left(\sqrt{\frac{E_{p}}{N_{0}}}\left(\Upsilon-\frac{s_{j}\left(\tau_{0}\right)}{\sqrt{E_{p}}}\right)\right)
\end{aligned}
$$

The Gaussian integral function, $Q(x)$, is simply the integral of a mean zero, unit variance Gaussian density function from $x$ to infinity. Here $s_{j}\left(\tau_{0}\right)$ is given in $(7)$ and an example of $s_{j}\left(\tau_{0}\right)$ is shown later in Figure 4.

The mean acquisition time, $E(T)$, can be computed using (10) by noting that the transition probabilities are inherently functions of the multipath channel parameters. Thus the quantity in (10) represents the mean acquisition time conditioned on the multipath parameters, $E(T \mid \mathbf{a}, \boldsymbol{\tau})$ where a and $\tau$ are $M \times 1$ vectors containing the multipath parameters $a_{m}$ and $\tau_{m}$. If the multipath channel is not known beforehand, then a statistical model will be required. The unconditional mean acquisition time for the problem at hand is

$$
E(T)=\frac{1}{T_{f}} \int_{0}^{T_{f}} \frac{1+\sum_{m=0}^{N-2} \prod_{n=0}^{m} p_{n}\left(\tau_{0}\right)}{1-\prod_{n=0}^{N-1} p_{n}\left(\tau_{0}\right)} \mathrm{d} \tau_{0}
$$

The search algorithm which determines the value of the $\varepsilon_{m}$ parameter in $s_{j}$ is examined in the next section for the 'ideal' case of unity detection probability, zero false alarm probability, and a consecutive number of bins which will terminate the search. This basically represents a 'no noise' or infinite SNR scenario.

\section{Serial Search Algorithms (No Noise)}

The 'search' variable will be denoted as $Y_{m}$ for $m=0,1, \cdots, \infty$ and examines bins in some predetermined order. Five different search algorithms will be investigated: A) linear search, B) truly random search, C) random permutation search, D) 'look and jump by $K$ bins' search and E) bit reversal search. The values of the 'true' hypotheses that will terminate the search are represented by the random variables $X_{1}, X_{2}, \cdots, X_{K}$, which are $K$ consecutive hypotheses for $K \in\{1,2, \cdots, N\}$. Note that $X_{k}$ will wrap from $N-1$ to 0 if need be. $M$ is the stopping time associated with finding the 'cluster' of bins $X_{1}, X_{2}, \cdots, X_{K}$ and is given as $M=\inf \left(m: Y_{m} \in\left\{X_{1}, X_{2}, \cdots, X_{K}\right\}\right)+1 . X_{1}$ is discrete uniform from 0 to $N-1$ and independent of $Y_{m}$ for all $m$. Thus we see that $X_{k}=X_{1}+k-1$ for $k=1,2, \cdots, K$ where the addition is modulo $N$.

\section{A. Linear Search}

For this particular search, $Y_{m}=m \bmod N$ for $m=0,1, \cdots, \infty$. The expected stopping time can be found as

$$
E(M)=\frac{(N-K)^{2}+(3 N-K)}{2 N}
$$

A plot of the normalized mean stopping time, $E(M) / N$, versus the parameter $K / N$ is shown in Figure 3 for $N=25$. The parameter $K / N$ is the fraction of the total search area occupied by terminating hypotheses. Of particular interest in the figure is that the linear search algorithm performs the poorest. A typical value of $N$ for the UWB frame time acquisition problem is roughly 10000. The linear search, as with all of the searches except the truly random search, gives the expected search time of $(N+1) / 2$ for $K=1$. Also, all the searches give a mean search time of 1 when $K=N$. 


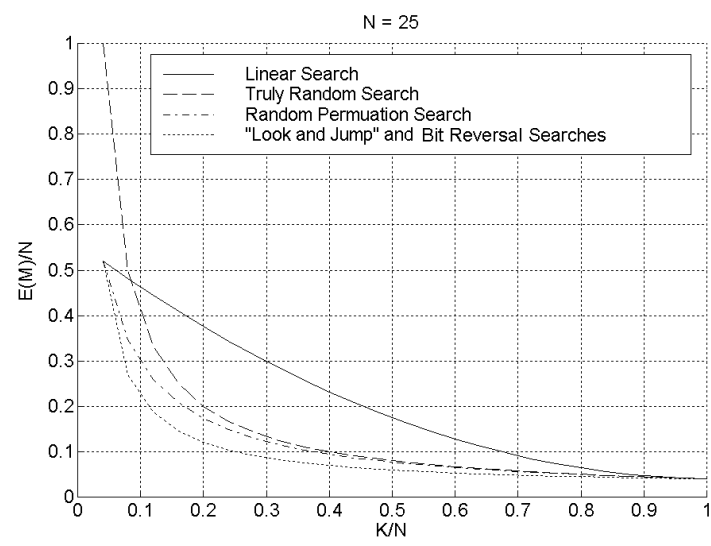

Fig. 3. Normalized mean stopping time for $N=25$ (Assumes unity detection probability, zero false alarm probability, and $K$ consecutive terminating search bins).

\section{B. Truly Random Search}

The truly random search is one in which the history of the previously searched bins is ignored. Thus the search variable, $Y_{m}$, is selected at random from $0,1, \cdots, N-1$ for each $m$. The expected stopping time can be computed as

$$
E(M)=\sum_{k=1}^{\infty} k \operatorname{Pr}(M=k)=\frac{N}{K}
$$

As can be seen in Figure 3 the truly random search actually performs better than the linear search when $K$ is only slightly larger than one.

\section{Random Permutation Search}

For this particular search strategy, the integers $\{0,1, \cdots, N-1\}$ are randomly permuted and the bins are searched according to this random permutation. More precisely, if $\sigma_{n}$ is a random permutation of $\{0,1, \cdots, N-1\}$ for $n=0,1, \cdots, N-1$, then the search random variable is simply $Y_{m}=\sigma_{m} \bmod N$ for $m=0,1, \cdots$. The mean stopping time is found to be:

$$
E(M)=\frac{N+1}{K+1}
$$

\section{D. 'Look and Jump by K Bins' Search}

The random permutation search algorithm previously discussed gives the mean stopping time, averaged over all permutations of the integers, $0,1, \cdots, N-1$. Since the linear search is one special case of such a permutation, and the mean stopping time of the random permutation search is lower than that of the linear search as seen in Figure 3 this reveals that certain permutations must exist that give an even lower mean stopping time. The 'Look and Jump by $K$ Bins' search described here is one such permutation, as is the search described in the next section.

The basic idea for the current search is as the name suggests, that is, starting in bin 0 , the search continues on to bin $K$, then to $2 K$, etc. The mean stopping time is found as

$$
E(M)=\frac{1}{2}\left(\frac{N}{K}+1\right)
$$

\section{E. Bit Reversal Search}

For UWB frame acquisition, the delay spread of the channel will not be known exactly or, quite possibly, at all. Also, due to the nature of the multipath channel, there will most likely not be $K$ consecutive bins which terminate the search but a cluster of bins, some with high probability and some with low probability of terminating the search. For this reason, an efficient search is desired which does not rely on any knowledge of $K$. The algorithm discussed in this section is such a search. In fact, the performance has been determined through computer simulation to be exactly the same as the 'Look and Jump' search of the previous section. Thus the mean stopping time of the bit reversal search is

$$
E(M)=\frac{1}{2} \cdot\left(\frac{N}{K}+1\right)
$$

The current search is described by assuming that $N$ is a power of 2, e.g., $N=2^{n}$ for $n$ a positive integer. The manner in which the bins are searched is then determined by 'bit reversing' the linear search variable. For example, the integers for an $N=16$ search can be represented in binary (base 2) as 0000 , 0001, 0010, 0011, 0100, $\cdots, 1101,1110,1111$. One permutation of these integers which maximizes the distance between observations is obtained by 'bit reversing' the binary representation, e.g., searching as per $0000,1000,0100,1100,0010, \cdots, 1011,0111$, 1111.

\section{UWB ACquisition Example}

The multipath channel parameters used for the example in this section are determined from the CLEAN algorithm on a measured waveform, i.e., see Figure 1. Two of the searches from the previous section are investigated, the linear search and the bit reversal search. For simplicity in dealing with this bit reversal search, the total number of bins is set to $N=2^{13}=8192$. To compensate for the current single-dwell example's lack of a verification phase, the input $E_{p} / N_{0}$ is set to a large value, namely $50 \mathrm{~dB}$. For the figures shown below, a normalized threshold of $\Upsilon=0.05$ is assumed.

For the mean correlator output shown in Figure 4, the transition probabilities of (11) can be computed. 


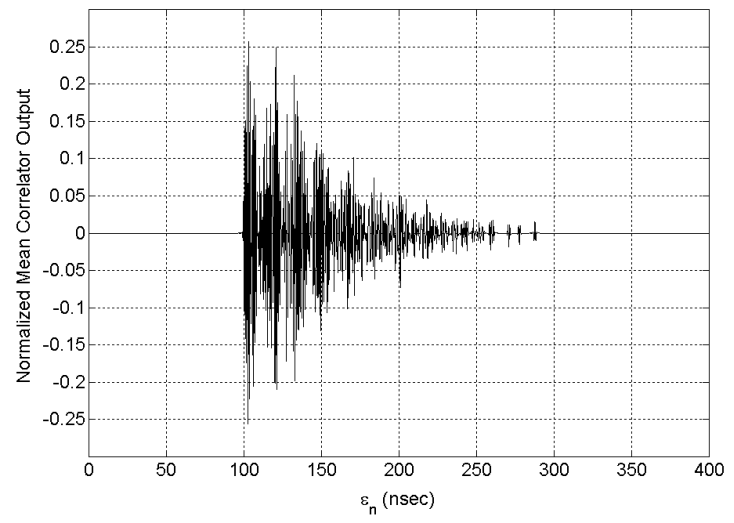

Fig. 4. Normalized mean correlator output, $s_{j} / \sqrt{E_{p}}$, for the reconstructed signal of Figure $1, \tau_{0}=100$ nsec, $T_{f}=1000$ nsec, $N=8192$ bins, and $\varepsilon_{n}=\frac{n}{N} \cdot T_{f}$ for $n=0,1, \cdots, N-1$.

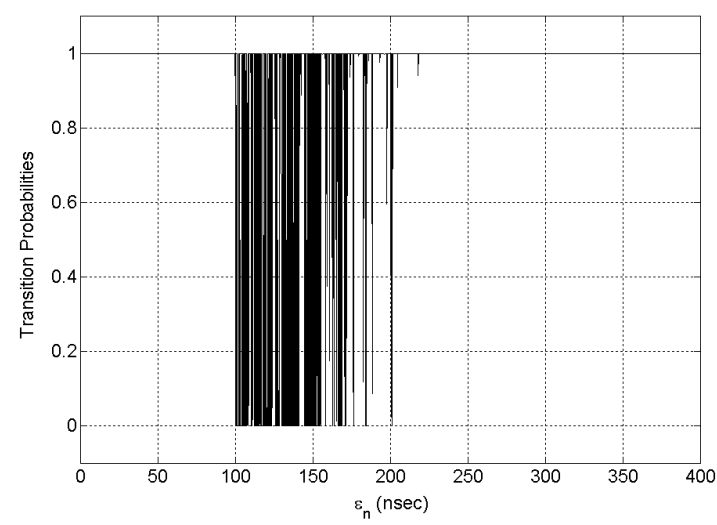

Fig. 5. Transition Probabilities, $p_{n}$, for the reconstructed signal of Figure $1, E_{p} / N_{0}=50 \mathrm{~dB}, \Upsilon=0.05, \tau_{0}=100$ nsec, $T_{f}=1000 \mathrm{nsec}, N=8192 \mathrm{bins}$, and $\varepsilon_{n}=\frac{n}{N} \cdot T_{f}$ for $n=0,1, \cdots, N-1$.

For illustrative purposes, $\tau_{0}$ is set to $100 \mathrm{nsec}$. The corresponding transition probabilities for the normalized threshold of $\Upsilon=0.05$ are shown in Figure 5 . Notice the that since the input SNR is large, the transition probabilities appear to be mostly limited to either 0 or 1 .

From (12) the expected number of observed search locations for the parameters at hand is found to be $E(T)=3246.6$ for the linear search, while the expected number for the bit reversal search is much smaller at $E(T)=28.7$. Thus the bit reversal search is roughly 113 times faster that the linear search for the multipath channel and system parameters currently considered. From Figure 5, it is noted that although the terminating hypotheses are not consecutive they can be viewed as almost consecutive over about 100 nsec. This implies that the $K / N$ parameter of section $\mathrm{V}$ is $100 / 1000=0.1$. The normalized mean acquisition times are seen to be $E(T) / N=3246.6 / 8192=0.396$ and $E(T) / N=$

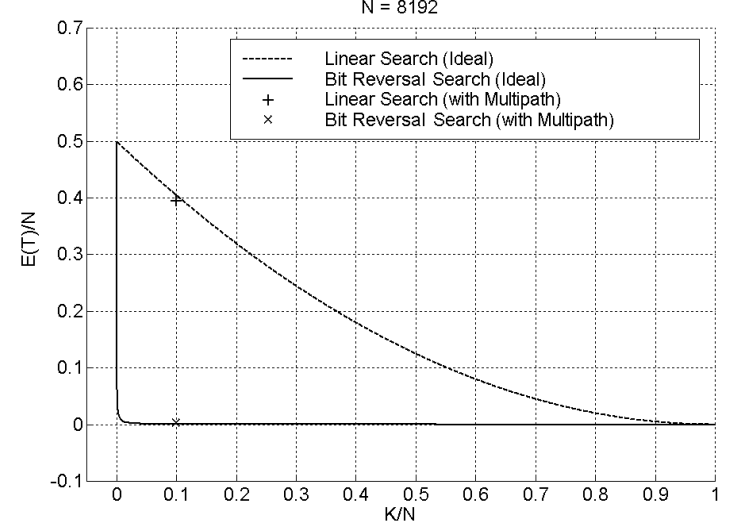

Fig. 6. Comparison of Normalized Mean Acquisition Time for the Multipath Channel versus 'Idealized' Results of Section $\mathrm{V}(N=8192)$.

$28.7 / 8192=0.0035$ for the linear and bit reversal searches, respectively. A plot of the results of section $\mathrm{V}$ are shown in Figure 6 for $N=8192$. The curves are for ideal detection and false alarm probabilities, as well as the assumption of consecutive terminating hypotheses as explained in section $\mathrm{V}$. These curves are labeled as '(Ideal)' searches. Also shown are the normalized mean acquisition times for the results computed in this section, labeled as the '(with Multipath)' searches.

\section{ViI. Concluding Remarks}

This paper has shown that when a large number of bins can terminate a search, a linear sweep of the bins is not a very efficient strategy. It has been shown that a specific permutation of the bin indices known as the bit reversal search can reduce the mean search time significantly, two orders of magnitude for the example considered here. Results from analyzing an 'ideal' search problem with perfect detection and false alarm probabilities and the assumption that a consecutive group of bins will result in termination were shown. These results closely approximated a high SNR acquisition example for an indoor multipath channel.

\section{REFERENCES}

[1] OSD/DARPA, Ultra-Wideband Radar Review Panal, Assessment of Ultra-Wideband (UWB) Technology, DARPA, Arlington, VA, 1990.

[2] M. Z. Win and R. A. Scholtz, "Ultra-wide bandwidth signal propagation for indoor wireless communications," in Proc. International Conf. Communications, Montreal, Canada, June 1997.

[3] R. A. Scholtz, "Multiple access with time-hopping impulse modulation," in Proc. IEEE Military Comm. Conf., Boston, MA, October 1993.

[4] M. Z. Win, Ultra-Wide Bandwidth Spread-Spectrum Techniques for Wireless Multiple-Access Communications, Ph.D. thesis, University of Southern California, Los Angeles, CA, May 1998. 